\title{
SOCIÉTÉ BOTANIQUE
}

DE FRANCE.

\section{SÉANCE DU 10 JUIN 1859. \\ PRÉSIDENGE DE M. DUCHARTRE.}

I. de Schœnefeld, secrétaire, donne lecture du procès-verbal de la séance du 27 mai, dont la rédaction est adoptée.

Par suite de la présentation faite dans la dernière séance, M. le Président proclame l'admission de :

M. Sevre (Jules), étudiant en médecine, rue Saint-Sulpice, 38, à Paris, présenté par MIM. Maurin et Damaskinos.

M. le Président annonce en outre une nouvelle présentation.

Lecture est donnée d'une lettre de M. Alph. Gacogne, qui remercie la Société de l'avoir admis au nombre de ses membres.

\section{Dons faits à la Société :}

$1^{\circ}$ De la part de M. Alph. Karr:

Les Guêpes, deux numéros.

$2^{\circ}$ En èchange du Bulletin de la Société :

Atti dell' I. R. Istituto veneto, juin 1859.

Pharmaceutical Journal and transactions, juin 1859.

L'Institut, juin 1859 , deux numéros.

M. J. Gay fait à la Société la communication suivante:

I. Alexandre Braun, dont j'avais appelé l'attention sur le mémoire de M. Tenore relatif à la transformation des fruits du Nymphcea alba, m'écrit qu'il a lu avec étonnement, mais aussi avec quelque incrédulité, les miracles que raconte l'auteur. "Son idée de la transformation d'un fruit mûr et déjà pourvu de graines en un tubercule végétant, me parait, dit-il, tout à fait inadmissible, et l'application qu'il en fait au Nymphaa Lotus, qui a des stolons comme le Fraisier, est sûrement inexacte; ou il a confondu avec

$$
\text { T. VI. }
$$


le Nymphcea alba une espèce différente qui portait des stolons, ou bien il a vu une chloranthie du Nymphceallalba, avec métamorphose du pistil en bourgeon foliaire; mais alors la fleur n'aurait point été normale; encore moins la métamorphose aurait-elle été précédée de la formation d'un fruit arrivé à maturité. n

M. de Schœnefeld, secrétaire, donne lecture de la communication suivante, adressée à la Société :

GLANES D'UN BOTANISTE, AVEG DES OBSERVATIONS SUR QUELQUES ESPÈCES

DU MIDI DE LA FRANGE, par M. Henri LORET.

NEUVIÈME PARTIE.

(Toulouse, 10 avril 1859.)

Hieracium pyrenaieum Jord. Obs, fragm. 7,1849 , p. 37 ; G. G. Fl. de Fr. t. II, p. 382, var, nobile Nob. (H. nobile G. G. Fl. de Fr, t. II, p. 376),

Je trouvai, le 21 août 1853, à Gèdre (Hautes-Pyrénées), un Hieracium que je pris pour l' $H$. pyrenaicum Jord., détermination qui fut sanctionnée plus tard par l'auteur de l'espèce. La même espèce se présenta à moi aux Eaux-Chaudes, en septembre 1855; et, sur un grand nombre de rochers, le long de la route des Eaux-Chaudes à Gabas. M, Gaston Sacaze me la donna alors des Eaux-Bonnes sous le nom d' $H$. nobile, en me disant que les nombreux botanistes qu'il voyait chaque année aux Eaux-Bonnes l'appelaient ainsi. Je lui fis observer qu'il y avait eu probablement double emploi dans la dernière Flore de France, et je crois avoir acquis en effet la certitude que les $H$. pyrenaicum Jord. et $\boldsymbol{H}$. nobile $\mathrm{G}$. G. sont deux synonymès qui se rapportent à la même espèce. La plante des Eaux-Bonnes ne diffère de celle de Gèdre que par l'absence des cils corollins, et, quoique ce caractère varie peu, j'ai eu néanmoins d'autres preuves de son défaut de constance, et peut-être y a-t-on attaché quelquefois trop de prix. M. Grenier n'ayant pu parler des akènes de son $\boldsymbol{H}$. nobile qu'il a décrit d'après un échantillon unique recueilli en juillet aux Eaux-Bonnes, je puis dire que les akènes d'un autre échantillon que j’ai trouvé également aux Eaux-Bonnes, dès 1846, et avant qu'il fût question de l' $H$. nobile, sont en tout conformes à ceux de la plante de Gedre $(H$. pyrenaicum Jord.). M. l'abbé de Lacroix (Bull. de la Soc. bot. de Fr. t. II, p. 458), parle d'un botaniste qui fit arracher, aux Eaux-Bonnes, six cents échantillons de l' $H$. nobile en une seule nuit. L'indiscrétion de ce botaniste reste, selon moi, sans compensation, car cette plante ne sera plus considérée comme très rare, lorsqu'une observation attentive aura démontré, comme j'en ai la convíction, que c'est à peine une variété de l'H. pyrenaicum publié antérieurement.

Hieraeium anglienm Fries (teste Grenier!). - Rochers granitiques. 


\section{$2 \mathrm{BHL}$ Biodiversity Heritage Library}

1859. "Séance Du 10 Juin 1859." Bulletin de la Société botanique de France 6, 385-386. https://doi.org/10.1080/00378941.1859.10829418.

View This Item Online: https://www.biodiversitylibrary.org/item/8630

DOI: https://doi.org/10.1080/00378941.1859.10829418

Permalink: https://www.biodiversitylibrary.org/partpdf/158205

\section{Holding Institution}

Missouri Botanical Garden, Peter H. Raven Library

\section{Sponsored by}

Missouri Botanical Garden

\section{Copyright \& Reuse}

Copyright Status: Public domain. The BHL considers that this work is no longer under copyright protection.

This document was created from content at the Biodiversity Heritage Library, the world's largest open access digital library for biodiversity literature and archives. Visit BHL at https://www.biodiversitylibrary.org. 